\title{
Evidence for an Io plasma torus influence on high-latitude Jovian radio emission
}

\author{
M. L. Kaiser and M. D. Desch \\ Laboratory for Extraterrestrial Physics, NASA Goddard Space Flight Center, Greenbelt, Maryland \\ M. E. Brown \\ Lunar and Planetary Laboratory, University of Arizona, Tucson
}

\begin{abstract}
We report the discovery with the Ulysses unified radio and plasma wave (URAP) instrument of features in the Jovian hectometer (HOM) wavelength radio emission spectrum which recur with a period about 2-4\% longer than the Jovian System III rotation period. We conclude that the auroral HOM emissions are periodically blocked from "view" by regions in the torus of higher than average density and that these regions rotate more slowly than System III and persist for considerable intervals of time. We have reexamined the Voyager planetary radio astronomy (PRA) data taken during the flybys in 1979 and have found similar features in the HOM spectrum. Contemporaneous observations by Brown (1994) show an [SII] emission line enhancement in the Io plasma torus that rotates more slowly than System III by the same amount as the HOM feature.
\end{abstract}

\section{Introduction}

Jupiter is a prolific source of nonthermal radio emissions. These emissions emanate from several distinct locations in the magnetosphere [see Kaiser, 1993, and references therein]. Most of these radio components are intensity modulated by the planetary 10-hour rotation period, and most of them also show a 26-day modulation imposed by the solar wind. One component, however, the narrowband kilometer wavelength (nKOM) emission, shows a modulation in its occurrence at some $3-8 \%$ slower than the planetary rotation period. This rate is consistent with the observed lack of corotation of the Io torus plasma in the vicinity of the nKOM source region, 8-10 $R_{J}$ near the centrifugal equator [Reiner et al., 1993a]. Sandel and Dessler [1988] cited earlier nKOM observations as one of several Io torus features that recur at a period of System III + $3 \%$, which they called System IV. We now present evidence of a similar modulation in another unrelated Jovian radio component, the hectometer wavelength (HOM) component. Since the HOM component is known to originate at high latitudes, this modulation at the System IV rotation period suggests a causal relationship between the auroral region and the Io torus.

Observations taken by the Unified Radio and Plasma Wave Experiment (URAP) aboard the Ulysses spacecraft during its approach to Jupiter in January and February 1992 are the primary data source used in this work. Supporting observations, described here for the first time, come from the data taken by the Voyager planetary radio astronomy (PRA) instruments during their encounters with Jupiter in 1979. All of the observations reported here were made from near Jupiter's equator.

The Ulysses URAP instrument consists of several receivers covering the band from $\mathrm{DC}$ to $1 \mathrm{MHz}$ (see full description by Stone et al. [1992a]). Of particular interest here is the high-

Copyright 1996 by the American Geophysical Union.

Paper number 95JA02389.

0148-0227/96/95JA-02389\$05.00 frequency portion of the radio astronomy receiver (RAR) which sweeps through 12 logarithmically spaced channels from 52 to $940 \mathrm{kHz}$ every $144 \mathrm{~s}$. The RAR is driven by a $72-\mathrm{m}$ dipole and a 6-m monopole. Ulysses is a spinning spacecraft with the long dipole in the spin plane and the monopole on the spin axis. Electrically combining the two antennas permits URAP to measure all four Stokes' parameters [Kraus, 1986] and the direction of arrival of the observed signals.

Each of the two Voyager instruments, fully described by Warwick et al. [1977], consists of two swept-frequency receivers. The lower band of these pairs of receivers is of interest here. During the era when the Voyagers were in the vicinity of Jupiter, the PRA low-band receivers swept the frequency range from 1.2 to $1326.0 \mathrm{kHz}$ in 70 linearly spaced steps every $6 \mathrm{~s}$ and measured the left- and right-hand circularly polarized power. The PRA receivers are driven by two $10-\mathrm{m}$ monopoles. Primarily owing to the longer antenna system, the URAP RAR is about a factor of 10 more sensitive near $1 \mathrm{MHz}$ than the PRA. The Voyager spacecraft are three-axis stabilized.

\section{Observations}

The HOM component was originally discovered by Earthorbiting spacecraft [Desch and Carr, 1974; Brown, 1974]. It consists of relatively smoothly varying emission of both left and right circular polarization in the band from about $300 \mathrm{kHz}$ to perhaps $2 \mathrm{MHz}$, with a spectral peak near $1 \mathrm{MHz}$. The HOM is only observed by spacecraft that are within several degrees of the Jovian equator and is not generally observed at high latitudes [Stone et al., 1992b]. The rotation profile consists of two regions of emission symmetrically situated approximately around the longitude of the north magnetic dipole, $210^{\circ}$ System III, as seen in Figure 1. Prior to the 1992 Ulysses flyby of Jupiter, it was believed that the source of HOM was at very high Jovian latitudes both north and south, with emission being generated via the electron cyclotron instability [ $W u$ and Lee, 1979] at very large angles to the magnetic field [Ladreiter and Leblanc, 1991]. The Ulysses flyby provided the first direct de- 


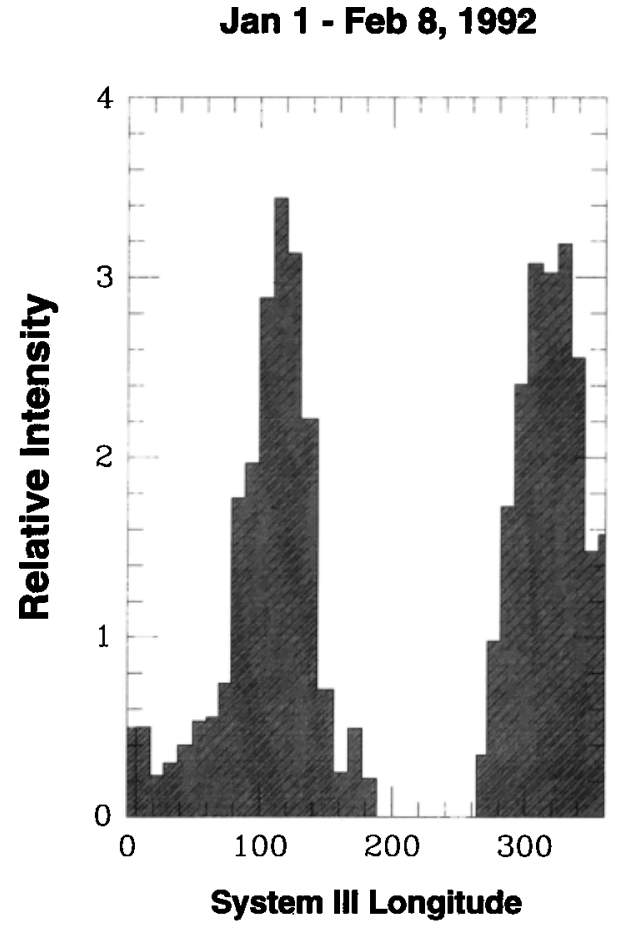

Figure 1. The average intensity of Jovian hectometer (HOM) wavelength radio emissions observed by the unified radio and plasma wave instrument (URAP) at $740 \mathrm{kHz}$ during the 100-rotation interval preceding the Ulysses flyby of Jupiter on February 8, 1992. The intensity is shown as a function of Ulysses' central meridian longitude.

terminations of the HOM source locations [Reiner et al., 1993b; Ladreiter et al., 1994]. URAP confirmed that HOM consists of two sources, north and south, but the latitudes of the sources were somewhat lower and the beaming angles somewhat less than expected.

Although the two peaks in the rotation profile shown in Figure 1 are approximately equal in intensity when averaged over a significant interval (about 100 Jovian rotations in the case of Figure 1), there is considerable variation from one rotation to the next. Plate 1 shows five consecutive 10-hour rotations from the URAP RAR in dynamic spectral format, where increasing relative intensity is color coded according to the bar at the bottom. The HOM can be seen in each panel above about $300 \mathrm{kHz}$ and in the central meridian longitude (CML) ranges of $50^{\circ}-150^{\circ}$ and $250^{\circ}-350^{\circ}$. The emission at lower frequencies near $200^{\circ}$ is broadband kilometer wavelength emission (bKOM) and is not of interest here. It can be seen that during this 5-rotation interval, the portion of HOM centered on the higher-longitude range is considerably more intense than that at lower longitudes. Furthermore, both parts of HOM exhibit considerable variation from one rotation to the next.

In order to examine these variations more carefully, we displayed a much longer interval of time, as shown in Plate 2. In this plate, data from the three highest-frequency channels of URAP $(540,740$, and $940 \mathrm{kHz})$ are averaged and displayed as relative color-coded intensity in a format of System III longitude versus arbitrary rotation number. Data for approximately 70 Jovian rotations during the month of January 1992, preceding the February 8, 1992, closest approach of Ulysses to Jupiter are shown. In order to correct for the intensity variation due to changing proximity to Jupiter, the levels have been adjusted to what an observer $0.1 \mathrm{AU}$ from the HOM source would observe. The variations between the two parts of HOM are evident; rarely are the lower- and higher-longitude portions of equal intensity. Rather, one part is dominant for several rotations followed by the other part.

The most striking and totally unexpected feature in Plate 2 is the tendency for intensity patterns to drift in longitude. The drifts are always toward higher longitudes with increasing rotation number. These drifts are particularly evident in the higher-longitude portion of HOM between January 1 and 15 but can also be seen at many other places in the plate. The drift rate differs from the planetary rotation rate by about $3 \%$, consistent with System IV, as shown. In retrospect, some suggestion of this drifting phenomenon can be seen in Plate 1, again in the higher longitude part of HOM, where the peak intensity tends to appear at successively higher longitudes in each panel.

Power spectral analysis of the entire 100-rotation (January 1 through February 8, 1992) data set for all 12 URAP RAR channels is shown in Figure 2, where relative spectral power is indicated by increasing darkness. The dominant periodicity is, quite naturally, at the System III rotation period of 9.925 hours. This periodicity is seen above $\sim 300 \mathrm{kHz}$ in the HOM component and below $\sim 200 \mathrm{kHz}$ in the bKOM component. The nKOM component can also be seen in the $100-$ to $200-\mathrm{kHz}$

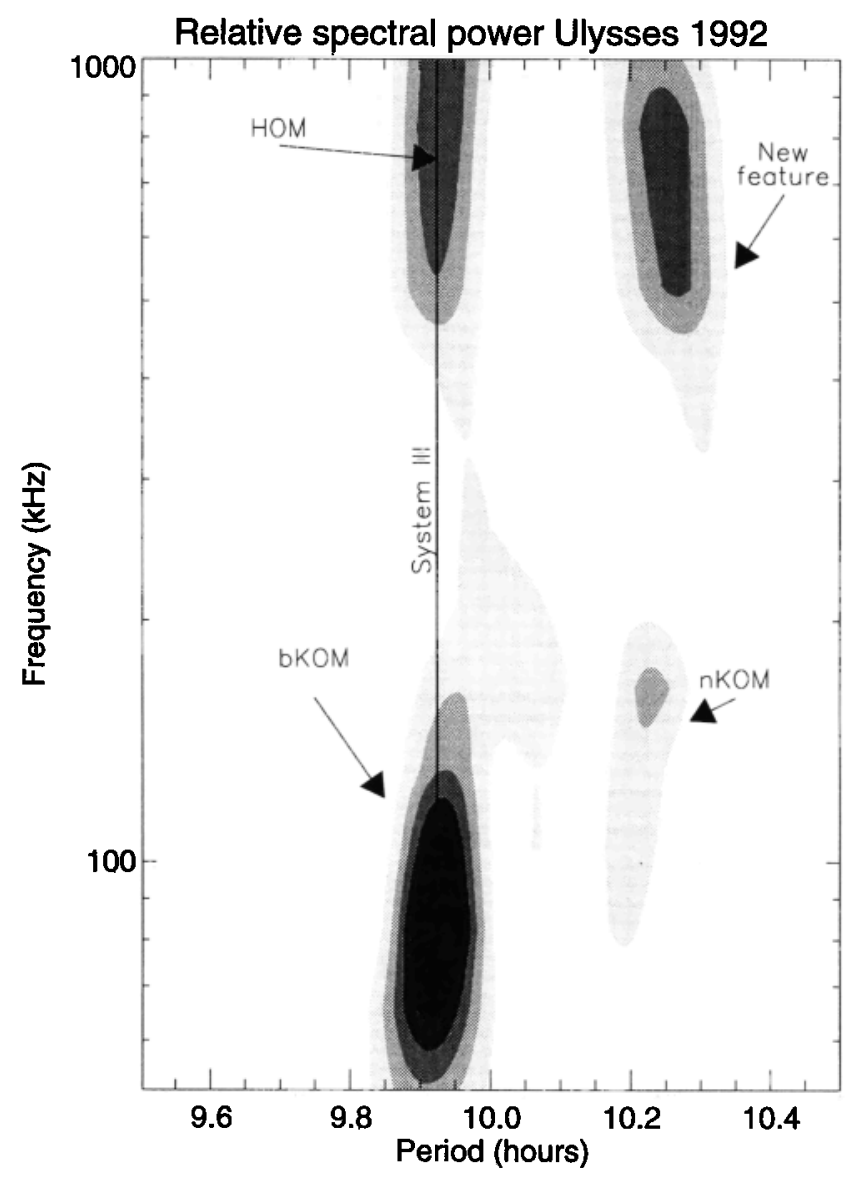

Figure 2. Relative spectral power for all 12 channels of the URAP high-frequency receiver during the 100-rotation interval preceding the February 8,1992 , Ulysses flyby of Jupiter. 

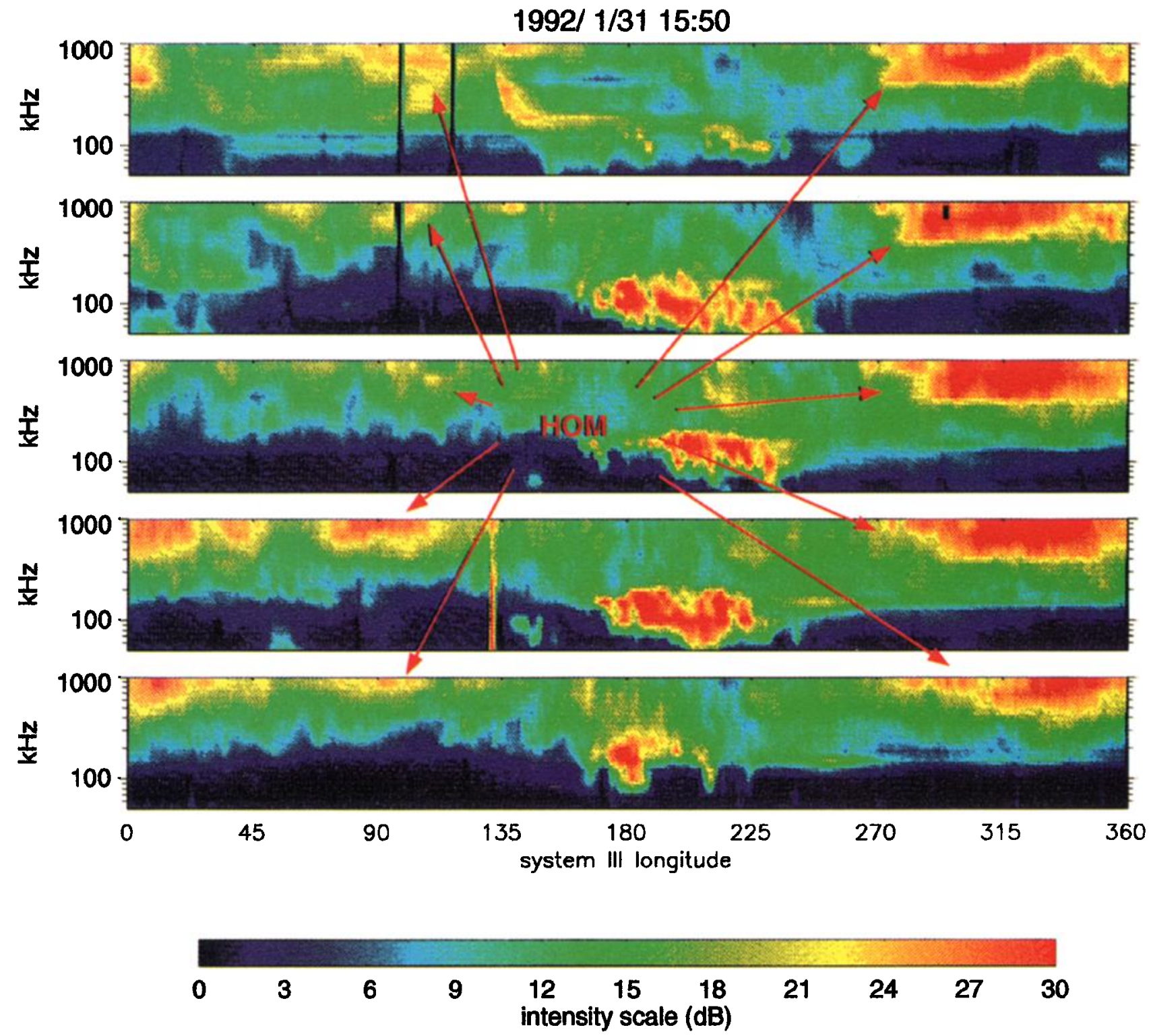

Plate 1. Data from the URAP high-frequency receiver for five consecutive Jovian rotations. Increasing intensity above the receiver background level is represented by the color bar shown at the bottom.

range with its characteristic System IV period. The region labeled "new feature" corresponds to the drifting emission shown in Plates 1 and 2. This feature is offset from the System III period by nearly the same amount as nKOM. There is also a suggestion from this figure that the feature does not extend above $\sim 1 \mathrm{MHz}$, although the upper frequency of the URAP RAR $(940 \mathrm{kHz})$ does not allow us to be certain.

Thus far, all data presented in Figure 1, Plates 1 and 2, and Figure 2 are from the URAP instrument taken during 1992. We have also reexamined the Voyager 1 and 2 PRA data from 1979 and find quite similar results. Figures $3 a$ and $3 b$ are the power spectral analysis schematics from the $\sim 100$-rotation intervals preceding the March 5, 1979, Voyager 1 flyby and the July 9, 1979, Voyager 2 flyby respectively, of Jupiter. The new feature is quite prominent in both figures, again appearing at the System IV period. The suggestion noted above, that this new feature does not extend above $1 \mathrm{MHz}$, is clearer in the Voyager data. We have examined the PRA data at frequencies above those shown in Figure 3 (the PRA receivers extend to $1.3 \mathrm{MHz}$ ) and find no evidence of the new feature.

It is not immediately obvious from Plates 1 and 2 and Figures 2 and 3 just what the exact nature of the new feature is. It could be an enhancement of one of the existing HOM "components" every 10.2 hours, or it could equally well be a periodic decrease of one of the components. Figure 4 lends some credence to the latter interpretation. Here the HOM intensity is plotted during a period of time when one of the HOM components starts to dominate in intensity over the other. During days $24-27$, there is an HOM intensity peak every $\sim 5$ hours with both of the HOM components, low longitude and high longitude, of roughly equal intensity. However, a transition takes place so that during days $29-36$ the 5 -hour periodicity is replaced with a 10-hour periodicity. It appears that one of the two peaks making up the 5-hour periodicity of days 24-27 has diminished considerably in intensity, whereas the other peak has remained approximately the same. 


\section{Ulysses/URAP}
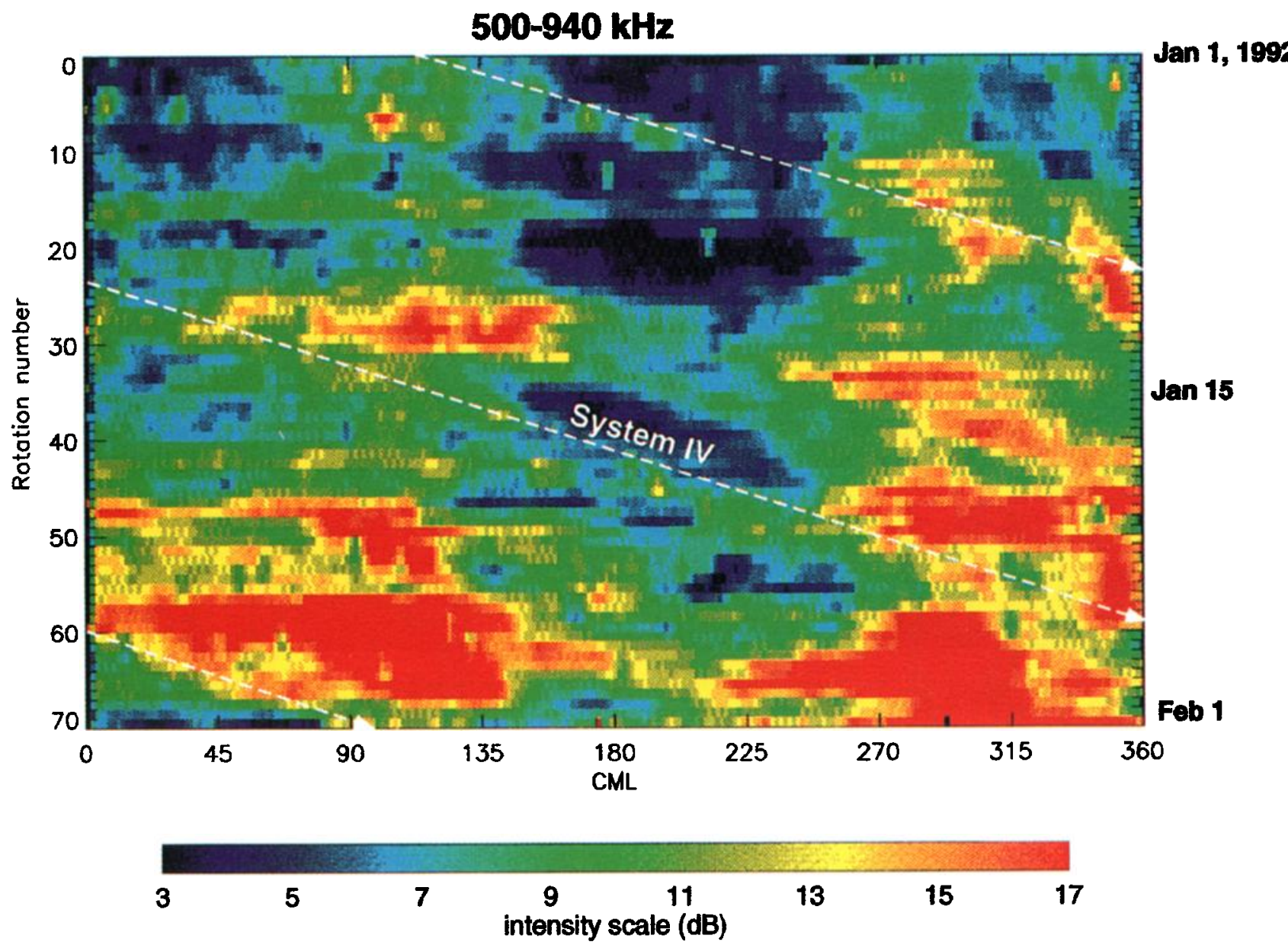

Plate 2. Relative intensities from the three highest-frequency channels of URAP during the month of January 1992, shown as a function of central meridian longitude and rotation number (arbitrary). The relative intensity is indicated by the color bar at the bottom. The diagonal lines serve two purposes. First, they show how System IV, in general, drifts relative to System III. Second, they show the actual position of the [SII] bright feature as a function of time.

\section{Discussion and Summary}

There have been reports over the past decade of optical features in the torus that rotated around Jupiter at approximately the System IV period. These reports include [SIII] observations of Roesler et al. [1984] and Woodward [1992], and Sandel and Dessler [1988] in a reexamination of the Voyager UVS observations at $685 \AA$.

During the 6-month interval roughly centered on the time of the Ulysses encounter with Jupiter, Brown [1994] made ground-based spectroscopic observations of the Io torus at 6716 and $6731 \AA$ [SII] and found a persistent bright feature that extended over a large radial range and rotated around Jupiter with a period equal to the System IV period. His analysis indicates that this feature is a density enhancement rather than a temperature variation and that the enhancement is about $50 \%$.

Brown [1994] gives an equation for the System III longitude of the bright spot,

$$
\lambda_{\text {III }}=24.6 \Delta t+120^{\circ}
$$

where $\Delta t$ is the time in days since 0 hour on January 1,1992 Returning to Plate 2, the actual location of the diagonal lines labeled "System IV" corresponds to the equation, thus indicating the location of the bright spot as a function of time. It must be remembered that the radio data in all figures are plotted as a function of the System III longitude of the observer, which is rarely the same as the longitude of the source region. The line representing the [SII] bright spot, however, is the longitude of the spot itself. Nevertheless, it can be seen that usually when the bright spot is near the central meridian, the HOM intensity seems to be diminished. Since the magnetic field lines passing through the bright spot at that instant do not generally also pass through the HOM source regions, we must conclude that the primary effect is on propagation of the HOM emission. We believe that the high-density spot must periodically refract HOM emission away from the observer's line of sight.

We have shown that there exists a long-lived feature in the Jovian HOM spectrum that rotates with the System IV. The feature manifests itself as a diminution in intensity. Optical 

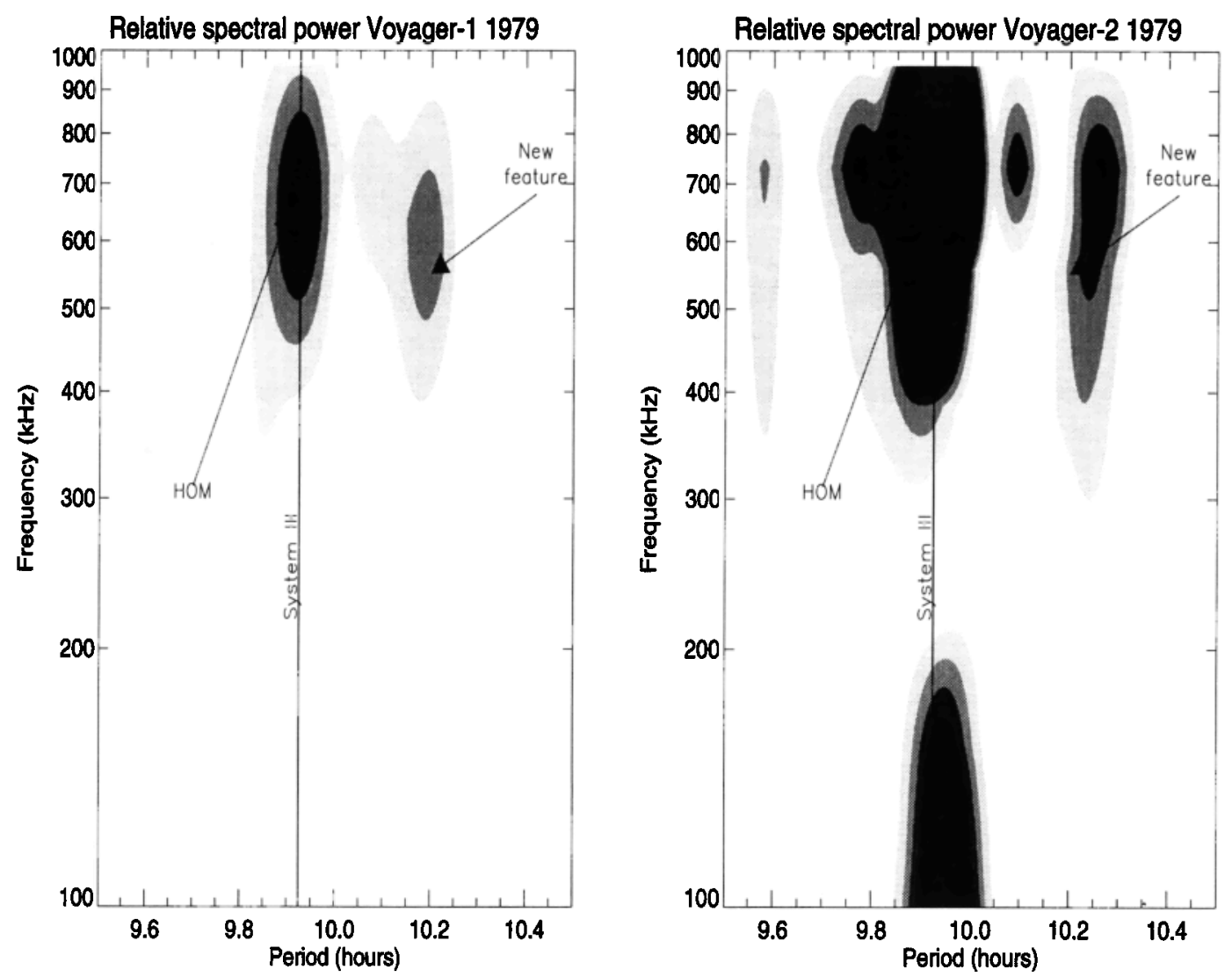

Figure 3. (a) Relative spectral power for the Voyager 1 PRA low-band receiver for the 100-rotation interval preceding the March 5, 1979, Jupiter encounter. (b) Similar to Figure 3a but for the Voyager 2 flyby on July 9, 1979.

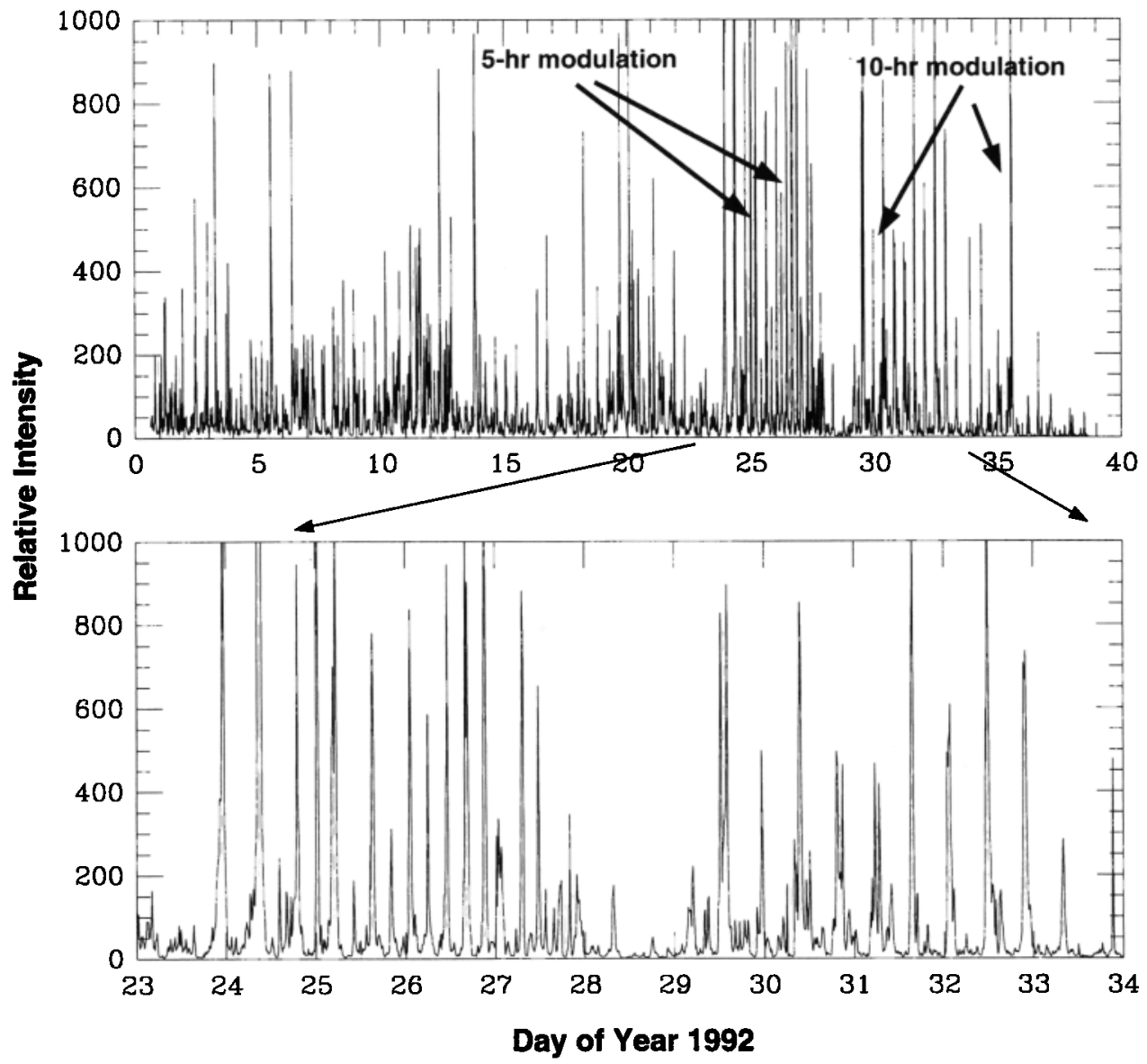

Figure 4. Top panel shows the relative intensity of Jovian HOM at $740 \mathrm{kHz}$ observed by URAP during the 100 -rotation interval preceding the February 8, 1992, Ulysses flyby of Jupiter. Bottom panel is an expanded view of days 23-34 of the top panel. 
spectroscopic observations by Brown [1994] during the same interval of time as a portion of the HOM observations show that there is a long-lived high-density region in the Io plasma torus that also rotates at the System IV period. The HOM feature and the torus high-density spot seem to be correlated such that, usually, when the spot is on or near the observer's central meridian, the HOM emission is partially or totally blocked.

These two new sets of observations, the HOM features and Brown's [1994] high-density region in the torus, add considerable weight to the contention by Sandel and Dessler [1988] and Yang et al. [1991] that Jupiter's magnetosphere has two independent repetition periods associated with it. It is not clear, however, which, if any, of the several mechanisms suggested by Sandel and Dessler to produce this dual periodicity is favored by the new observations.

Perhaps the most intriguing aspect of this story is the persistence of the feature. The feature is evident in both radio and ultraviolet observations from the Voyagers in 1979, in groundbased spectroscopic observations during the mid-1980s and early 1990s, and in the Ulysses radio data. This suggests that there is a permanent, high-density region in the Io plasma torus which rotates at the System IV period.

Acknowledgment. The Editor thanks two referees for their assistance in evaluating this paper.

\section{References}

Brown, L. W., Spectral behavior of Jupiter near $1 \mathrm{MHz}$, Astrophys. J., 194, L159, 1974.

Brown, M. E., The structure and variability of the Io plasma torus, Ph.D. dissertation, Univ. of Calif. at Berkeley, 1994.

Desch, M. D., and T. D. Carr, Decametric and hectometric observations of Jupiter from the RAE-1 satellite, Astrophys. J., 194, L57, 1974.

Kaiser, M. L., Time-variable magnetospheric radio emissions from Jupiter, J. Geophys. Res., 98, 18,757, 1993.
Kraus, J. D., Radio Astronomy, 2nd ed., Cygnus-Quasar, Powell, Ohio, 1986.

Ladreiter, H. P., and Y. Leblanc, The Jovian hectometric radiation: An overview after the Voyager mission, Ann. Geophys., 9, 784, 1991.

Ladreiter, H. P., P. Zarka, and A. Lecacheux, Direction finding study of Jovian hectometric and broadband kilometric radio emissions: Evidence for their auroral origin, Planet. Space Sci., 42, 919, 1994.

Reiner, M. J., J. Fainberg, R. G. Stone, M. L. Kaiser, M. D. Desch, R. Manning, P. Zarka, and B.-M. Pedersen, Source characteristics of Jovian narrow-band kilometric radio emissions, J. Geophys. Res., 98, $13,163,1993 a$.

Reiner, M. J., J. Fainberg, and R. G. Stone, Source characteristics of Jovian hectometric radio emissions, J. Geophys. Res., 98, 18,767, $1993 b$.

Roesler, F. L., F. Scherb, and R. J. Oliverson, Periodic intensity variation in [SIII] $9531 \AA$ emission from the Jupiter plasma torus, Geophys. Res. Lett., 11, 128, 1984.

Sandel, W. R., and A. J. Dessler, Dual periodicity of the Jovian magnetosphere, J. Geophys. Res., 93, 5487, 1988.

Stone, R. G., et al., The unified radio and plasma wave investigation, Astron. Astrophys. Suppl. Ser., 92, 291, 1992a.

Stone, R. G., et al., Ulysses radio and plasma wave observations in the Jupiter environment, Science, 257, 1524, 1992b.

Warwick, J. W., J. B. Pearce, R. G. Peltzer, and A. C. Riddle, Planetary radio astronomy experiment for the Voyager missions, Space Sci. Rev., 21, 309, 1977.

Woodward, R. C., Sulfur emission and periodicity of the Jupiter plasma torus in 1988, Ph.D. dissertation, Univ. of Wisc.-Madison, 1992.

Wu, C. S., and L. C. Lee, A theory of the terrestrial kilometric radiation, Astrophys. J., 230, 621, 1979.

Yang, Y. S., A. J. Dessler, and B. R. Sandel, Is System IV independent of System III?, J. Geophys. Res., 96, 3819, 1991.

M. E. Brown, Lunar and Planetary Laboratory, University of Arizona, Tucson, AZ 85721. (e-mail: mbrown@lpl.arizona.edu)

M. D. Desch and M. L. Kaiser, Laboratory for Extraterrestrial Physics, NASA Goddard Space Flight Center, Greenbelt, MD 20771. (email: kaiser@lepmlk.gsfc.nasa.gov)

(Received May 1, 1995; revised July 31, 1995; accepted July 31, 1995.) 